\title{
Improved Electronic Image Stabilisation Based on Image Mosaic and Grey Projection
}

\author{
Shaoqing Tian \\ Business Administration Postdoctoral Station, Harbin University of Commerce, Harbin 150000, \\ China \\ Email: tiansq1@163.com
}

\begin{abstract}
In the study of electronic image stabilisation algorithm, it is found that image processing has the problem of missing image information. According to the TRIZ problem analysis theory, a method is presented in this paper, which improves the results of electronic image stabilisation through the combination of image mosaic and grey projection. Based on the processing results of the grey projection algorithm, the reference frame and the current frame are image spliced to obtain a continuous sequence of missing images. The simulation experiment shows that the method is feasible and efficient.
\end{abstract}

Keywords: Image Mosaic, Electronic Image Stabilisation, TRIZ, Grey Projection

\section{INTRODUCTION}

Smart devices are being used more and more in people's daily lives, such as iPhone, Android, UAVs and Google Glass. These devices all have one or more cameras, with sophisticated camera functions and as a result, a large number of images are produced, including continuous and discontinuous, static and dynamic, local and panoramic, blurred and clear images. These images appear due to the functions, performance or operation of the devices or the users' own needs. Although these images keep people's beautiful moments or wonderful processes, not all of them can be easily obtained because some or all of them have problems, whether manmade or non-manmade. Therefore, it is necessary to study the problems existing in the process of image acquisition, including image stabilisation, panorama image acquisition and rapid graphics processing, so that people can better use smart devices to capture their desired images.

Based on these problems, this paper presents an electronic image stabilisation method based on image mosaic and grey projection to address image losses after electronic image stabilisation. In addition, a main contribution of this paper is using image mosaic to improve the processing results of the electronic image stabilisation algorithm. Finally, we verify the algorithm based on grey image projection.

\section{RELATED LITERATURE REVIEW}

There are many related research results although few scholars and experts have contributed in this regard. In terms of the Electronic Image Stabilisation (EIS) technology, the research trend has always maintained stable, indicating that this technology has entered a mature stage, so currently scholars in this field mainly focus on combining multiple algorithms and application. Specific researches are as follows.

Zhao [1] described the visual characteristics of human eye, and proposed a kind of inter-frame motion amplitude statistics algorithm based on the video quality evaluation algorithm and human's visual characteristics. Being in high consistency with the subjective evaluation results, this algorithm can effectively evaluate the performance of the electronic image stabilisation algorithm. Fan [2] studied the mine car camera system. In order to quickly and accurately stabilise the jittered images obtained from the system, an electronic image stabilisation algorithm based on downsampling grey projection is proposed. This method is superior to the traditional grey projection algorithm in terms of accuracy and computation time. Gong [3] described the shortcomings of the grey projection algorithm, such as single grey value and poor contrast, and proposed an electronic image stabilisation algorithm based on block grey projection, which can improve the peak signal-to-noise ratio by $21.5 \%$. Bai [4] studied the application of the block grey projection algorithm in satellite assembly and proposed an electronic image algorithm based on partial grey projection to ensure that the robot system could output stable and coherent satellite assembly images. Yuan [5] analyzed the slowness and inaccuracy of the traditional estimation methods under video jitter and proposed a motion estimation method combining grey scale and block matching to improve the computational speed and estimation accuracy of the global motion estimation method. Ji [6] described the problem that the feature points in the foreground moving objects would 
seriously affect the operational vector accuracy, and proposed a video stabilisation method based on background feature point matching. This method improves the video smoothness after electronic image stabilisation. CZ You [7] studied the time consumption by the grey projection algorithm during image stabilisation. In the case where the image stabilisation is not affected, this method distinguishes the images, uses the improved algorithm to solve the grey projection vector, and employs the mean filter to allow the global motion vector to realize the motion compensation.

From the existing researches, it is not difficult to find that electronic image stabilisation technology is mainly used to address the instability of continuous images, with the output being a series of images. There is no research on the improvement of algorithm processing. Therefore, this study focuses on improving the electronic image stabilisation technology, which will have a certain contribution to this field.

\section{PRINCIPLES OF EIS AND TRIZ}

\subsection{Principle of EIS}

The basic principle of EIS technology is to obtain the offset of the jittered image through calculation, and then compensate the image so that the jittered image and the nonjittered image can be overlapped, as shown in Figure 1.

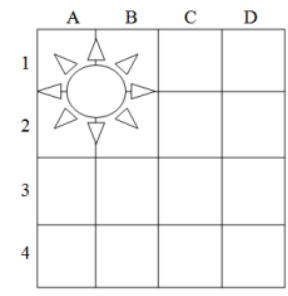

A. Non-jittered image

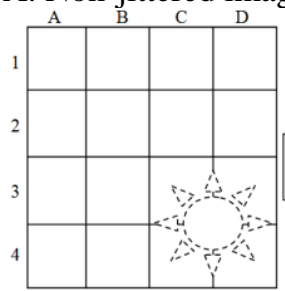

C. Jittered image

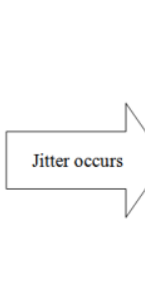

ge
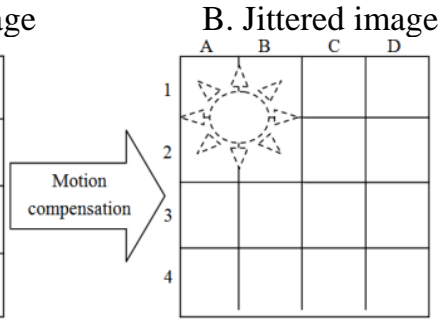

D. Motion Compensated
Figure 1. Basic principle of electronic image stabilisation

Figure 1A is an image with no jitter among a series of successive images, where each small grid represents one pixel. After the jitter occurs, the "sun" at A1A2B1B2 is shifted to C3C4D3D4 in Figure 1B, and the continuous playback of the image produces fuzziness. In order to make the image stably and clearly displayed, it is necessary to move the image in the reverse direction, as shown in Figure $1 \mathrm{C}$ and 1D. The jittered image is shifted by two pixels to the left and two pixels upward, so that the jittered image and the non-jittered one are overlapped to result in a clear and stable image sequence.

EIS is a way for us to stabilise images through digital image processing. It includes digital image acquisition through electronic devices like DV, DC and camera, digital image processing and digital image output.

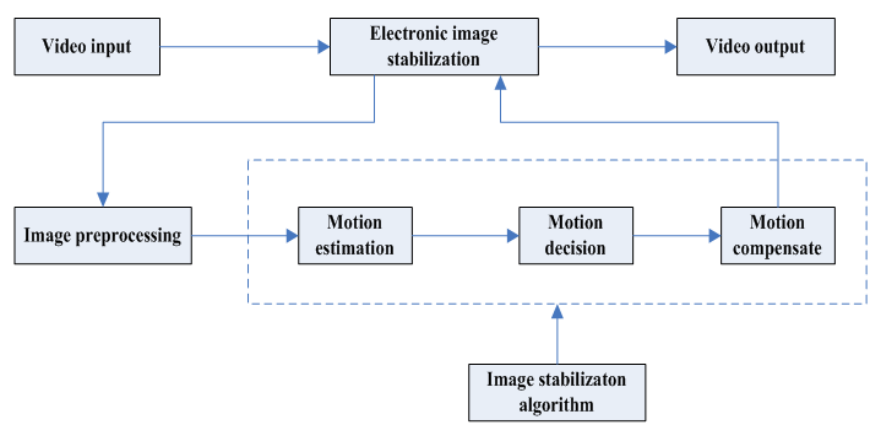

Figure 2. Flow chart of electronic image stabilisation

\subsection{Principle of TRIZ}

TRIZ was developed in 1946 by GS Altshuller, an inventor of the former Soviet Union. Altshuller discovered that any field of product improvement, technological change, innovation is same as the biological system, where there is a process of producing, growing, maturing, aging and dying. Under his leadership, research institutes, universities and enterprises in the former Soviet Union formed the TRIZ research community, analysed nearly 2.5 million high-level invention patents in the world, summarised the regular patterns of various technological developments and evolutions. Based on a set of innovative theories and rules of technological and physical contradictions, a comprehensive theoretical system was established, composed of various methods and algorithms, for solving technical problems, and then it was innovated and developed into the TRIZ theoretical system based on the principles and rules in multiple disciplines and fields.

The core idea of modern TRIZ theory [8] is mainly reflected in three aspects: be it a simple product or a complex technical system, the development of its core technology follows the objective laws and evolution patterns. The continuous solutions to the technical problems and conflicts is the driving force behind this evolutionary process. The ideal objective of technological system development is to use as few resources as possible to achieve as many functions as possible.

Innovation is, in its most general sense, a process of creatively discovering and solving problems. The TRIZ theory is powerful because it provides a systematic theoretical and methodological tool for people to discover and solve problems creatively. The TRIZ theoretical system includes the following aspects.

(1) Innovative thinking method and problem analysis method. TRIZ provides scientific methods on how to systematically analyse problems, such as the multi-screen method. For complex problems, it includes a scientific problem analysis and modelling method - object-field analysis, which can help quickly identify the core problem and find where the fundamental contradiction lies.

(2) Evolution of technical systems. Aiming at the evolvement rule of technical system, based on a large number of patent analyses, the TRIZ theory concludes eight basic evolution rules. Using these evolutionary laws, we can analyse and confirm the technical status of current products, predict future trends, and develop competitive new products.

(3) Principle of technical conflict resolution. Different inventions often follow common rules. The TRIZ theory summarizes these common rules into 40 inventive principles. Based on these inventive principles and specific engineering 
contradictions, we can find concrete solutions to specific technical conflicts.

(4) Standard solution to the problem of innovation. According to the different characteristics of object-field models for specific problems, there are corresponding standard model processing methods, including the dressing and transformation of the model and the addition of material and field, and so on.

(5) Invention problem solving algorithm ARIZ. This is mainly for complex technical problems and conflicts and related components of the system are not clear. It is a noncomputational logic process involving a series of transformations and redefinitions of the initial problem, so as to gradually and deeply analyses and transforms the problem until it is solved.

(6) Knowledge base built on the engineering principles in physics, chemistry and geometry, etc. A knowledge base based on the analysis of millions of invention patents in the fields of physics, chemistry and geometry can provide abundant program sources for technological innovation.

Compared with the traditional trial-and-error method and other innovative methods like brainstorming, the TRIZ theory has distinct characteristics and advantages. It successfully reveals the inherent laws and principles of creating invention and focuses on clarifying and emphasising the contradictions existing in the system rather than avoiding them. Its goal is to completely resolve the contradictions and obtain the ultimate ideal solution instead of making compromises, and it studies the entire design and development process based on the technological evolution rule, rather than performs random behaviours.

Practice has proved that using the TRIZ theory can greatly accelerate the process where people create inventions and obtain high-quality innovative products. It can help people systematically analyse the situation of the problem, quickly find the essence or contradiction of the problem and accurately identify the directions to explore the problem without missing many possibilities, and also help people break through their thinking barriers, adjust their mindset, carry out logical and non-logical systematic thinking from new perspectives, and predict future development trends based on the technological evolution rule to help them develop new competitive products.

\section{METHOD OF IMPROVEMENT FOR EIS AND PIM}

The improved method mainly includes two parts - one is the electronic image stabilisation based on the grey projection algorithm (GPA), and the other, the image mosaic method.

\subsection{Problem analysis based on TRIZ theory}

At present, the TRIZ theory has a wide range of applications, mainly in such fields as machinery, electronics, construction, food and chemical production. In the software design and algorithm research, the TRIZ theory has rarely been used. However, the TRIZ theory will bring great convenience to future research if it can be successfully applied to the study of algorithms. Below is how to analyse the problem scenario by using the TRIZ problem analysis and solving tools.

(1) Analysis of images and algorithm

When people record a video, they aim to shoot a specific object or scene, but part of it is unnecessary information. For example, when we monitor a forest path, pedestrians walking on the path is not necessary information. The grey projection algorithm is to use the reference image and the grey projection of the current image and calculate the offset through a certain correlation, and then reverse the effect of the image to stabilise it. In the absence of pedestrians, the reference image has little difference from the grey projection curve of the current image, so the algorithm works well, but when pedestrians walk or stay away from the monitor, the grey projection value of the whole picture will change obviously, and the accuracy of the algorithm will decrease because the algorithm does not need to read this information in the image. This requires improving the algorithm to enhance its adaptability.

(2) Extraction of technical conflicts

Considering the actual situation, the projection algorithm needs two images - the reference frame and the current frame. There is no pedestrian in the reference frame while there are in the current frame. There is a way to remove the pedestrians that affect the grey projection from the image, but it also removes the background information, making the information of the image incomplete and directly affecting the validity of the image. This has formed a technical contradiction.

(3) Selection of inventive principles

According to the description of the conflict, the unnecessary information in the image needs to be removed, but the whole image information needs to remain complete. Based on the 39 common engineering parameters listed in TRIZ, two pairs of parameters can be chosen - \#6 area of non-moving object and \#24 loss of information, \#31 harmful side effects and \#24 loss of information. And then establish the TRIZ conflict matrix:

A. \#6 and \#24 have two inventive principles: \#30 flexible membranes or thin film and \#16 partial or overdone action;

B. \#31 and \#24 have three inventive principles: \#10 prior action, \#21 rushing through and \#29 pneumatic or hydraulic construction;

(4) Inventive principles and actual combination

From the listed inventive principles, it can be found that only \#16 partial or overdone action in a and \#10 prior action in $\mathrm{b}$ can be used as the directions for innovation.

First, according to TRIZ's explanation of \#16, if it is difficult to obtain $100 \%$ of a desired effect, achieve somewhat more or less to greatly simplify the problem.

To remove pedestrians from the image, if the image is processed with a less-than-desired effect, then it is very hard to get rid of all the unnecessary information; and if the image is processed with more than the desired effect, pedestrian information can be completely removed, but it is not certain whether this more than the desired effect is appropriate. It can be seen from the image that with or without pedestrians, the forest information remains unchanged, but the road changes due to the changes in the greyscale information of pedestrians. We might as well directly remove the road from the image so that no matter whether there is a pedestrian or not, we cannot see it from the greyscale. How to remove the road from the image is a problem to be solved. It can be observed from the image that the forest is mainly green, and that the road is grey, so we use colours to distinguish the forest and the road. In this way, we can completely get rid of the road, and also achieve the expectation.

Second, according to TRIZ's explanation of \#10, carry out all or part of the required action in advance and arrange 
objects so they can go into action in a timely matter and from a convenient position.

This principle is exactly in line with the requirements of image pre-processing for electronic image stabilisation. This processing exceeds its intended effect, but it can be used as a pre-processing method for the image.

Finally, it can be seen that the TRIZ theory helps us find the solution to the problem - pre-processing the image more than intended.

\subsection{Improved electronic image stabilisation method based on grey projection}

The image can present different information if the image is graded according to the greyscale, and the different grey level planes can reflect the different information that the greyscale image cannot show [9].

An image of visible light contains a lot of information, where the greyscale information reflects the darkness of each pixel. The images obtained by visible light devices are usually saved based on the RGB colour model. If you want to obtain the grey information of each pixel from the RGB image, it is generally necessary to calculate the R, G, and B values corresponding to each pixel of the image through the $\mathrm{Eq}(1)$.

$$
G(x, y)=(R+G+B) / 3
$$

where $G(x, y)$ is the grey value of the point ( $\mathrm{x}, \mathrm{y})$ (in the decimal system), and R, G, and B are the values of red, green, and blue, respectively.

After obtaining the grey value, the grey level plane can be extracted from the grey image. The specific steps are described below:

Step 1: Convert the grey value of each pixel in the image from decimal to binary, and divide the value into eight grey levels. For example, if the grey value is 255 (decimal), then the grey value can be expressed as 11111111 (binary). The conversion equation is as follows:

$$
G(x, y)=b_{K-1} 2^{K-1}+b_{K-2} 2^{K-2}+\cdots+b_{1} 2+b_{0}
$$

where $b_{k}=1$ or 0 and $\mathrm{K}$ is the grey level (the default value of $\mathrm{K}$ is equal to 8 ).

Step 2: After obtaining the binary grey value, extract only the value of each bit corresponding to the binary, and obtain the grey bitmap from the newly formed image. The conversion equation is as follows:

$$
g_{k}(x, y)=b_{k-1} 2^{k-1} \quad 1 \leq k \leq K
$$

where, $g_{k}(x, y)$ is the $\mathrm{k}$-th level grey value.

On this basis, perform electronic image stabilisation to calculate the offset more accurately. The EIS based on GPA includes the following 4 steps.

Step 1: Convert the image into a grey image.

Step 2: Obtain the row and column projection value through grey projection. The calculation formula is as follows.

$$
\operatorname{row}(i)=\sum_{j=1}^{n} \operatorname{pic}(i, j) ; \operatorname{column}(j)=\sum_{i=1}^{m} \operatorname{pic}(i, j)
$$

$\operatorname{row}(i)$ is the row projection value on line $i \cdot \operatorname{column}(j)$ is the column projection value on series $j \cdot \operatorname{pic}(i, j)$ is the grey value at the point of $(i, j)$.

Step 3: Obtain the image jitter offset through correlation operation. This formula is as follows.

$$
C(w)=\sum_{j=1}^{n}[\operatorname{column}(j+w-1)-\operatorname{column}(M+j)]^{2} ; 1 \leq w \leq 2 M+1
$$

$C(w)$ is the correlation value. $\mathrm{M}$ is the maximum extent of jitter detected in the image. $w_{\min }$ is the $w$ when $C(w)$ is minimum.

Now, the column and row offsets can be computed through the following formula. The column offset is computed, so is the row offset.

motion $_{c}=M+1-W_{\min }$

Step 4: Move the jittered image by motion $_{r}$ and motion m $_{c}$ in the opposite direction, and then we can acquire the stable image.

\subsection{Image mosaic method based on SIFT A algorithm}

The main idea of this paper is to improve the processing results of electronic image stabilisation by using image mosaic technology. Here the SIFT algorithm is used to match images [10]. The specific steps are as follows:

Step 5: Feature point extraction

Convert the image obtained through Step 4 into a 32-bit grey image and subject it to cubic interpolation and Gaussian blurring. On this basis, construct the Gaussian Pyramid and the Gaussian Differential Pyramid. Then check the extreme points and calculate the scale of the feature vector, direction of the feature, and the descriptor.

Step 6: Feature matching

Extract the external feature vectors, including scaling, rotation, brightness changes, etc., from the reference frame image and the processed image. Use the Euclidean distance of the key point feature vector to determine the similarity of the key points in the two images.

Step 7: Image registration

Use the typical RANSAC algorithm in the matching point pair purification process.

Step 8: Image fusion

Decompose the image to be processed in accordance with the Laplace Pyramid. Splice images at different scales into the image at the corresponding scale by using different weighting functions and splicing area widths. Finally, combine the images at different scales into one complete image.

Through these 8 steps, we can obtain a complete image sequence with electronic image stabilisation. The feasibility of this method will be verified in the next experiment.

\section{EXPERIMENTS AND VERIFICATION}

The experiment carried out in this paper is a simulation test. The experimental data came from pictures manually shot 
with iPhone6 camera. The panoramic image mosaic algorithm used was the one mentioned in reference [11] and the electronic image stabilisation algorithm used was the grey projection image stabilisation algorithm. The experimental environment was the MATLAB7 simulation platform under Windows7.

First, the grey projection image stabilisation algorithm is used to obtain a stabilised image sequence 1, as shown in Figure 3.

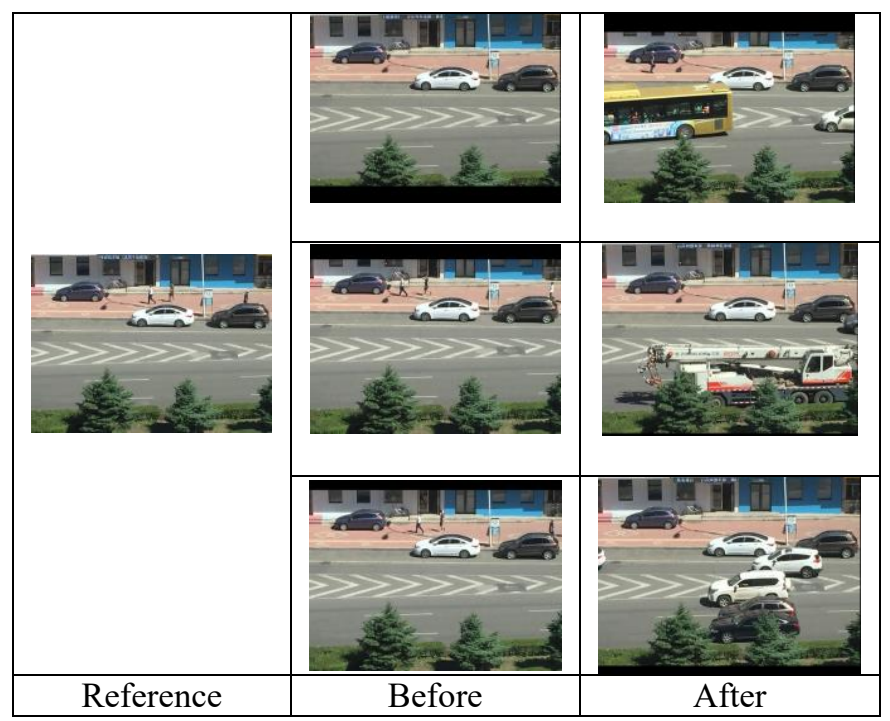

Figure 3. Image sequence before and after GPA processing

Then, the stabilised image in the image sequence 1 is paired with the reference-frame image to form a complete image, and the image sequence 2 is output, as shown in Figure 4.

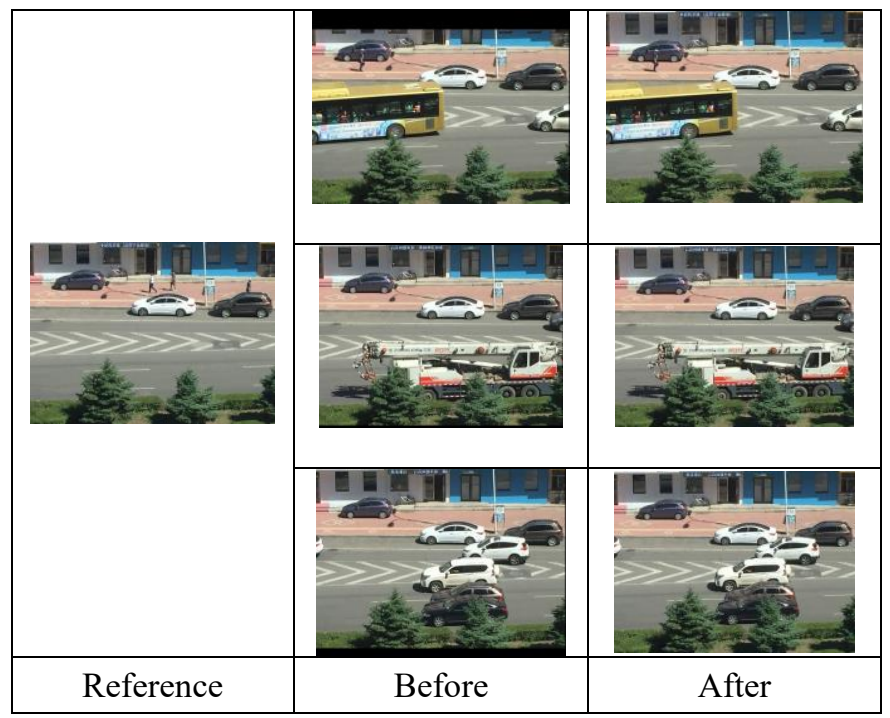

Figure 4. Image sequence after panoramic image mosaic process

\section{CONCLUSIONS}

There are many researches on the electronic image stabilisation algorithm based on grey projection. However, no one has proposed or studied how to address the missing image information to improve the processing effect. In light of this, according to the TRIZ theory for problem analysis, this paper studies a method combining PIM and GPA and finds that the missing part of the GPA-processed image can be supplemented by PIM. Experiments show that this method is effective and feasible.

\section{ACKNOWLEDGMENT}

The authors are grateful to the constructive and helpful comments of the anonymous referees. This study is supported by the Harbin Science and Technology Bureau Innovation Talent Special Foundation (Youth Reserve Talent Plan Category, 2016RAQXJ045), the Dr. Scientific Research Foundation of Harbin University of Commerce (2016BS17) and the Postdoctoral Research Support Program of Harbin University of Commerce (2017BSH013).

\section{REFERENCES}

[1] Zhao D.F., Zhang L. (2017). Performance evaluation method of non - reference electronic image stabilization algorithm based on motion amplitude statistics, China Science and Technology Papers, Vol. 12, No. 08, pp. 929-934.

[2] Fan Y.P., Guo Z., Zhang R. (2017). Study on electronic image stabilization algorithm based on down-sampling gray-scale projection, Mining Industry Automation, No. 4, pp. 22-27.

[3] Gong J.M., Xu J.C., Xing R.P. (2017). Based on block gray scale projection of electronic image stabilization algorithm, Journal of Xi'an University of Post and Telecommunications. No. 2, pp. 60-64.

[4] Bai F., Zhang X.J., Zhang M.L., Sun L.Y. (2016). Application of stereoscopic algorithm based on partition gray scale projection in satellite assembly, China Mechanical Engineering, N0. 2, pp. 195-200.

[5] Yuan W., Gao Y.Q., Wu J.L. (2016). Study on video stabilization of UAV based on gray projection and block matching, Radio Engineering, No. 2, pp. 19-22.

[6] Ji S.J., Lei Y.M., Zhu M. (2016). Video stabilization based on background feature point matching, Journal of Jilin University (Engineering Science), No. 6, pp. 2116-2122.

[7] You C.Z., Chen G.X. (2015). Improved gray-scale projection electronic image stabilization algorithm, Guilin University of Electronic Technology, No. 2, pp. 152-155.

[8] Wang N.H., Tian S.Q. (2011). TRIZ theory is used to improve based on gray projection algorithm for electronic image stabilization, Applied Mechanics \& Materials, Vol. 58-60, pp. 2017-2022.

[9] Huang Y.L., Tian S.Q. (2013). Forest fire smoke recognition based on gray bit plane technology, International Journal of Signal Processing, Image Processing and Pattern Recognition, Vol. 6, No. 6, pp. 309-320.

[10] Hao F., Wang C.M., Li X.B. (2012). Panoramic image stitching algorithm based on SIFT feature matching, Silicon Valley, Vol. 5, No. 22, pp. 176+132.

[11] Matthew B., David G.L. (2007). Automatic panoramic image stitching using invariant features, International Journal of Computer Vision, Vol. 74, No. 1, pp. 59-73. 